\title{
ON THE SKOLEM PROBLEM AND SOME RELATED QUESTIONS FOR PARAMETRIC FAMILIES OF LINEAR RECURRENCE SEQUENCES
}

\author{
ALINA OSTAFE AND IGOR E. SHPARLINSKI
}

\begin{abstract}
We show that in a parametric family of linear recurrence sequences $a_{1}(\alpha) f_{1}(\alpha)^{n}+\ldots+a_{k}(\alpha) f_{k}(\alpha)^{n}$ with the coefficients $a_{i}$ and characteristic roots $f_{i}, i=1, \ldots, k$, given by rational functions over some number field, for all but a set of elements $\alpha$ of bounded height in the algebraic closure of $\mathbb{Q}$, the Skolem problem is solvable, and the existence of a zero in such a sequence can be effectively decided. We also discuss several related questions.
\end{abstract}

\section{INTRODUCTION}

1.1. Motivation and background. We recall that a linear recurrence sequence $\left(u_{n}\right)_{n=1}^{\infty}$ of order $k$ over a field $\mathbb{K}$ is a sequence which satisfies a relation of the form

$$
u_{n+k}=A_{k-1} u_{n+k-1}+\ldots+A_{0} u_{n}, \quad n \geqslant 0,
$$

with some constants $A_{0}, \ldots, A_{k-1}, u_{0}, \ldots, u_{k-1} \in \mathbb{K}$; we refer to [18] for a background on recurrence sequences.

It is well known by the celebrated Skolem-Mahler-Lech Theorem, see for example [18, Theorem 2.1], that the set of zeros of a linear recurrence sequence $\left(u_{n}\right)_{n=0}^{\infty}$, that is, the set of $n \in \mathbb{N}$ such that $u_{n}=0$, is the union of a finite set with finitely many arithmetic progressions. The famous Skolem problem is a problem of decidability of the existence of such a zero. This problem remains widely open for most of the interesting fields $\mathbb{K}$ of characteristic zero, including $\mathbb{K}=\mathbb{Q}$ for $k \geqslant 5$, a brief outline of the current state of affairs is given by Sha [33]. In particular, although there are very good, uniform in all parameters and depending only on $k$, bounds on the number of zeros, see [3], there are no effectively computable bounds on the index $n$ of a possible zero $u_{n}=0$.

2010 Mathematics Subject Classification. 11B37, 11D61.

Key words and phrases. Skolem problem, perfect power, linear recurrence sequence. 
Here we address a parametric version of this problem for a family of linear recurrence sequences and for all but finitely many values of the parameter for which the specialised sequence is not degenerate we give a bound on the largest possible zero. In particular, the Skolem problem is effectively decidable for all but a set of values of bounded height of the parameter $\alpha \in \overline{\mathbb{Q}}$.

More precisely, we now recall that if the characteristic polynomial

$$
\Psi(Z)=Z^{k}-A_{k-1} Z^{k-1}-\ldots-A_{0}
$$

has $k$ distinct roots $\lambda_{1}, \ldots, \lambda_{k}$ then for any sequence $\left(u_{n}\right)_{n=1}^{\infty}$ as in (1.1) there are some $\mu_{1}, \ldots, \mu_{k}$ in an algebraic extension of $\mathbb{K}$ such that

$$
u_{n}=\sum_{i=1}^{k} \mu_{i} \lambda_{i}^{n}, \quad n \geqslant 0 .
$$

Linear recurrence sequences of this type are called simple. Some of the results on zeros of linear recurrence sequences in our arguments, are also known for arbitrary sequences, for example, those of [33], but some are known only under this condition, for example, those of [2].

In the case of $\mathbb{K}=\mathbb{C}$, the charactestic roots $\lambda_{i}, i=1, \ldots, k$, of the largest absolute value, that is, with

$$
\left|\lambda_{i}\right|=\max \left\{\left|\lambda_{1}\right|, \ldots,\left|\lambda_{k}\right|\right\}
$$

are called dominant and play a major role in investigating various Diophantine properties of linear recurrence sequences.

We recall that a sequence $\left(u_{n}\right)_{n=1}^{\infty}$ satisfying (1.1) is called degenerate if one of the ratios $\lambda_{i} / \lambda_{j}, 1 \leqslant i<j<k$, is a root of unity. We call a sequence non-degenerate otherwise.

It is very well-known that the Skolem problem for a degenerate sequence $u_{n}$ can be reduced to a series of the Skolem problems for finitely many non-degenerate sequences of the form $u_{n h+j}, j=0, \ldots, h-1$, where $h$ is bounded only in terms of the degree $\left[\mathbb{Q}\left(\lambda_{1}, \ldots, \lambda_{k}\right): \mathbb{Q}\right]$, see [5]. Hence we are mostly interested in non-degenerate sequences.

Here we study the case when both the coefficients $\mu_{i}$ and the roots $\lambda_{i}$ are rational functions of a parameter $\alpha \in \overline{\mathbb{Q}}$. This scenario is close to that of Amoroso, Masser and Zannier [2], see also [24, Proposition 2.2]. In particular, we also appeal to some of the results from [2], however our method is based on a different argument.

More precisely, given two vectors of rational functions

$$
\mathbf{a}=\left(a_{1}, \ldots, a_{k}\right), \mathbf{f}=\left(f_{1}, \ldots, f_{k}\right) \in \overline{\mathbb{Q}}(X)^{k},
$$


over the algebraic closure $\overline{\mathbb{Q}}$ of $\mathbb{Q}$ we consider linear recurrence sequences of the form

$$
F_{n}(X)=\sum_{i=1}^{k} a_{i} f_{i}^{n}, \quad n \geqslant 0 .
$$

We give a bound for the largest zero in (all but finitely many) specialisations of this sequence such that the new sequence is not degenerate, see Theorem 2.1. This is based on recent results of Pakovich and the second author [32] about finiteness of points on curves that lie on the unit circle, a bound for the largest zero in a linear recurrence with at most two dominant roots due to Sha [33] and a result of Amoroso, Masser and Zannier [2, Theorem 1.5] giving an upper bound for the height of zeros of polynomials of the form (1.2). We then couple our result with a general $\mathrm{ABC}$ theorem for polynomials [11, Theorem 12.4.4] to give a lower bound on the degree of the splitting field of $F_{n}$ over $\mathbb{Q}$ when $a_{i}, f_{i}$ are all polynomials, see Corollary 2.5 , which gives an explicit form of [2, Example 2].

We also look at complex numbers which are zeros of two functions of the form (1.2), see Theorem 2.9. This is closely related to studying the greatest common divisors of elements of two sequences defined by such functions. This point of view has been introduced by Ailon and Rudnick [1] for polynomials of the form $f^{n}-1$ and $g^{m}-1, n, m \geqslant 1$, and further developed in recent works [16, 26, 27, 31,32] for different other sequences. In turn, this builds on number field analogues of this problem, initiated by Bugeaud, Corvaja and Zannier [14] for sequences of the form $a^{n}-1$ and $b^{m}-1, n, m \geqslant 1$, where $a, b \in \mathbb{Z}$ are multiplicatively independent, and further extended in many works, including generalisations to $S$-units [15].

When we restrict to the set $\mathbb{U}$ of all roots of unity only, we can say more about the arithmetic structure of $F_{n}(\alpha), n \geqslant 1$, as defined in (1.2), that is, for all but finitely many $\alpha \in \mathbb{U}$ we prove that the set of $n \in \mathbb{N}$ such that $F_{n}(\alpha)$ is a perfect $m$ th power either contains an arithmetic progression, or it is finite, see Theorem 2.7. We conclude the paper with an independent result, Theorem 2.12, which says that a sequence of rational functions is a linear recurrence sequence if and only if for uncountably many $\alpha \in \mathbb{C}$ the specialisations of the sequence have the same property.

\section{Main Results}

2.1. Skolem problem for parametric families. We use $M_{\mathbb{K}}$ to denote a complete set of inequivalent absolute values on a number field $\mathbb{K}$, 
normalized so that the absolute Weil height $h: \overline{\mathbb{Q}} \rightarrow[0, \infty)$ is defined via $v$-adic valuations $\|\alpha\|_{v}$ as follows

$$
h(\alpha)=\sum_{v \in M_{\mathbb{K}}} \frac{\left[\mathbb{K}_{v}: \mathbb{Q}_{v}\right]}{[\mathbb{K}: \mathbb{Q}]} \log \max \left\{\|\alpha\|_{v}, 1\right\},
$$

where $\mathbb{K}$ is any number field containing $\alpha$ and $\mathbb{K}_{v}$ is the completion of $\mathbb{K}$ with respect to the absolute value $v$. See $[11,22,36,41]$ for further details on absolute values and height functions.

To formulate our results we need to recall that a finite Blaschke product is a rational function $B(z) \in \mathbb{C}(z)$ of the form

$$
B(z)=\zeta \prod_{i=1}^{n}\left(\frac{z-a_{i}}{1-\bar{a}_{i} z}\right)^{m_{i}},
$$

where $a_{i}$ are complex numbers in the open unit disc $\{z \in \mathbb{C}:|z|<1\}$, the exponents $m_{i}, i=1, \ldots, n$, are positive integers, and $|\zeta|=1$.

A rational function $Q(z)$ of the form $Q(z)=B_{1}(z) / B_{2}(z)$, where $B_{1}$ and $B_{2}$ are finite Blaschke products, is called a quotient of finite Blaschke products.

We say that a pair of rational functions $\left(g_{1}(X), g_{2}(X)\right) \in \mathbb{C}(X)^{2}$ is exceptional if they can be decomposed as

$$
g_{1}=Q_{1} \circ g \quad \text { and } \quad g_{2}=Q_{2} \circ g
$$

for some quotients of finite Blaschke products $Q_{1}$ and $Q_{2}$ and rational function $g$. Otherwise we say that $\left(g_{1}(X), g_{2}(X)\right)$ is a non-exceptional pair.

We recall that Blaschke products preserve the unit circle and thus come naturally in our investigation of $\alpha \in \overline{\mathbb{Q}}$ for which the corresponding specialisation of $F_{n}(X)$ given by (1.2) has at least three characteristic roots with the same modulus, that is, with

$$
\left|f_{r}(\alpha)\right|=\left|f_{s}(\alpha)\right|=\left|f_{t}(\alpha)\right|,
$$

for some $1 \leqslant r<s<t \leqslant k$, which implies

$$
\frac{\left|f_{s}(\alpha)\right|}{\left|f_{r}(\alpha)\right|}=\frac{\left|f_{t}(\alpha)\right|}{\left|f_{r}(\alpha)\right|}=1 \text {. }
$$

We see that if $g_{1}$ and $g_{2}$ are polynomials and thus have no finite poles, relations (2.2) are possible only if $n=1$ and $a_{1}=0$ in (2.1), in which case we obtain

$$
g_{1}=g^{m_{1}} \quad \text { and } \quad g_{2}=g^{m_{2}}
$$

for some $g \in \mathbb{C}[X]$ and integers $m_{1}, m_{2} \geqslant 0$.

Since as we have mentioned, the Skolem problem for degenerate sequences is easily reducible to the case of non-degenerate sequences, it 
motivates us to define for the parametric family (1.2) the exceptional set of $\alpha \in \overline{\mathbb{Q}}$ such that $f_{i}(\alpha) / f_{j}(\alpha)$ is not a root of unity for some $1 \leqslant i<j \leqslant k$. It is also convenient to eliminate the roots of $a_{i}(X)$ and $f_{i}(X), 1 \leqslant i \leqslant k$. That is, we define the set

$\mathfrak{E}_{\mathbf{a}, \mathbf{f}}=\left\{\alpha \in \overline{\mathbb{Q}}: f_{i}(\alpha) / f_{j}(\alpha)\right.$ is a root of unity for some $1 \leqslant i<j \leqslant k$ or $a_{i}(\alpha)=0$ or $f_{i}(\alpha)=0$ for some $\left.1 \leqslant i \leqslant k\right\}$.

Using [36, Theorem 3.11] we immediately derive that elements of $\mathfrak{E}_{\mathbf{a}, \mathbf{f}}$ are of bounded height.

Our first result is the following:

Theorem 2.1. Let $a_{i}, f_{i} \in \overline{\mathbb{Q}}(X), i=1, \ldots, k$, be nonzero rational functions of degree at most $d$ such that $f_{i} / f_{j}$ is non-constant for any $1 \leqslant i<j \leqslant k$. We also assume that for any $1 \leqslant r<s<t \leqslant k$, the pairs of rational functions $\left(f_{s} / f_{r}, f_{t} / f_{r}\right)$ are non-exceptional. Let the sequence $\left(F_{n}\right)_{n=0}^{\infty}$ be defined by (1.2). Then for all but at most $2 d^{2} k(k-1)(k-2) / 3$ elements $\alpha \in \overline{\mathbb{Q}} \backslash \mathfrak{E}_{\mathbf{a}, \mathbf{f}}$ any zero $n \in \mathbb{N}$ of the equation

$$
F_{n}(\alpha)=0
$$

satisfies

$$
n \leqslant \exp \left(C D_{\alpha}^{4}\right)
$$

where $D_{\alpha}$ is the degree of the Galois closure of $\mathbb{Q}(\alpha)$ over $\mathbb{Q}$ and $C$ is an effective constant which depends only on $a_{1}, f_{1}, \ldots, a_{k}, f_{k}$.

Remark 2.2. We note that the condition that $f_{i}(\alpha) / f_{j}(\alpha)$ are not roots of unity for any $i \neq j$ is necessary for any bound on $n$ as otherwise the sequence can have infinitely many zeros. However, if this is the case, then by the celebrated Skolem-Mahler-Lech Theorem, see for example [18, Theorem 2.1], the set of zeros is the union of a finite set with finitely many arithmetic progressions, and by [5] these arithmetic progressions can be effectively determined, while our method allows to estimate the elements in the remaining finite set.

It is natural to ask whether a generalisation of Theorem 2.1 to parametric $S$-unit equations is possible, see Section 8 for more details and an exact question.

Theorem 2.1, immediately implies the following:

Corollary 2.3. Let $a_{i}, f_{i} \in \overline{\mathbb{Q}}[X], i=1, \ldots, k$, be as in Theorem 2.1. Let $\mathcal{A}_{D}$ be the union of all Galois fields of degree at most $D$ over $\mathbb{Q}$. Then there is an effectively computable constant $C_{0}$ which depends only on $a_{1}, f_{1}, \ldots, a_{k}, f_{k}$ such that for all but at most $12 d D^{2} k^{2}+2 d^{2} k^{3} / 3$ elements $\alpha \in \mathcal{A}_{D}$, any zero $n \in \mathbb{N}$ of the equation (2.3) satisfies

$$
n \leqslant \exp \left(C_{0} D^{4}\right) .
$$


Combining Theorem 2.1 with a result of Amoroso, Masser and Zannier [2, Theorem 1.5] (see Lemma 3.4), we also derive the following.

Corollary 2.4. Let $a_{i}, f_{i} \in \overline{\mathbb{Q}}[X], i=1, \ldots, k$, be as in Theorem 2.1. Then there exists a finite set $\mathcal{E} \subseteq \overline{\mathbb{Q}}$ such that for all roots $\alpha$ of $F_{n}$, $n \geqslant 1$, with $\alpha \notin \mathcal{E} \cup \mathfrak{E}_{\mathbf{a}, \mathbf{f}}$ the degree $D_{\alpha}$ of the smallest Galois extension over $\mathbb{Q}$ containing a satisfies

$$
D_{\alpha} \geqslant c(\log n)^{1 / 4}
$$

where $c$ is an effective constant depending only on $a_{1}, f_{1}, \ldots, a_{k}, f_{k}$.

Finally Theorem 2.1 coupled with a result of Fuchs and Pethö [21, Corollary 3.1] (see Lemma 3.8) yields the following result.

Corollary 2.5. Let $a_{i}, f_{i} \in \overline{\mathbb{Q}}[X], i=1, \ldots, k$, be as in Theorem 2.1 and such that $\operatorname{gcd}\left(a_{1} f_{1}, \ldots, a_{k} f_{k}\right)=1$. Then the splitting field $\mathbb{L}_{n}$ of the polynomial $F_{n}$ defined by (1.2) is of degree

$$
\left[\mathbb{L}_{n}: \mathbb{Q}\right] \geqslant c_{0}(\log n)^{1 / 4},
$$

where $c_{0}$ is an effective constant depending only on $a_{1}, f_{1}, \ldots, a_{k}, f_{k}$.

Note that Corollary 2.5 provides an explicit version of the claim that

$$
\left[\mathbb{L}_{n}: \mathbb{Q}\right] \rightarrow \infty
$$

as $n \rightarrow \infty$ given in [2, Example 2].

The bound for $n$ in Theorem 2.1 depends on the degree of the specialisation $\alpha$. We would like to obtain a bound which is independent of $\alpha$, when we restrict $\alpha$ to special subsets of $\overline{\mathbb{Q}}$, such as the set of all roots of unity. This in particular would imply that the set

$$
\left\{\alpha \in \overline{\mathbb{Q}}: \alpha^{n}=1, F_{m}(\alpha)=0 \text { for some } n, m \geqslant 1\right\},
$$

is finite, where $F_{m}$ is defined by (1.2).

More generally, since $G_{n}(X)=X^{n}-1, n \geqslant 1$, also defines a linear recurrence sequence, we would like to generalise the above situation and formulate the following problem.

Question 2.6. Given two simple linear recurrence sequences $\left(F_{n}\right)_{n=1}^{\infty}$, $\left(G_{m}\right)_{n=1}^{\infty}$ over $\mathbb{C}(X)$, prove that, under certain conditions, the set

$$
\mathcal{L}\left(F_{n}, G_{m}\right)=\left\{\alpha \in \mathbb{C}: F_{n}(\alpha)=G_{m}(\alpha)=0 \text { for some } n, m \geqslant 1\right\}
$$

is finite.

This would extend the result of [2] which already gives the boundedness of heights of $\alpha \in \overline{\mathbb{Q}}$ that satisfy only one such relation over $\overline{\mathbb{Q}}$ 
We answer Question 2.6 for binary sequences in Section 2.3 below, which is a direct consequence of [9, Theorem 2]. We also prove a more general result which holds only over $\overline{\mathbb{Q}}$, see Theorem 2.9 .

2.2. Perfect powers in specialisations at roots of unity. We now restrict $\alpha$ to the set $\mathbb{U}$ of all roots of unity. In this case, for all but finitely many $\alpha \in \mathbb{U}$ we can effectively answer some other questions about $F_{n}(\alpha)$ besides vanishing. We illustrate this approach on the case of perfect powers.

We use $\mathbb{Z}_{\overline{\mathbb{Q}}}$ to denote the set of all algebraic integers in $\overline{\mathbb{Q}}$.

We say that $\vartheta \in \mathbb{Z}_{\overline{\mathbb{Q}}}$ is a perfect $m$ th power if for some $\rho \in \mathbb{Q}(\vartheta)$ we have $\vartheta=\rho^{m}$.

For a polynomial $f \in \mathbb{C}[X]$, we use $\bar{f}$ to denote the complex conjugate polynomial, that is, the polynomial obtained from $f$ by conjugating all its coefficients.

Theorem 2.7. Let $a_{i}, f_{i} \in \mathbb{Z}_{\overline{\mathbb{Q}}}[X], i=1, \ldots, k$, be nonzero polynomials of degree at most $d$ such that for any $1 \leqslant r<s<t \leqslant k$, the pairs of rational functions $\left(f_{s} / f_{r}, f_{t} / f_{r}\right)$ are non-exceptional. We also assume that for $1 \leqslant i \neq j \leqslant k$ the polynomials $f_{i}(X) \overline{f_{i}}(Y)-f_{j}(X) \overline{f_{j}}(Y)$ do not have any factor of the form $X^{r} Y^{s}-u$ or $X^{r}-u Y^{s}$ where $u$ is a root of unity. Let the sequence of polynomials $\left(F_{n}\right)_{n=0}^{\infty}$ be defined by (1.2). Then for all but at most $d^{2}\left(2 k^{3} / 3+22 k^{2}\right)$ elements $\alpha \in \mathbb{U} \backslash \mathfrak{E}_{\mathbf{a}, \mathbf{f}}$ for any $m \geqslant 1$ the set of $n \in \mathbb{N}$ such that $F_{n}(\alpha)$ is a perfect mth power either contains an infinite arithmetic progression $m \ell+r, \ell=1,2, \ldots$, with some $r \in\{0, \ldots, m-1\}$ or is finite and its size can be effectively bounded.

Remark 2.8. We note that by a result of Lang [25] the set $\mathbb{U} \cap \mathfrak{E}_{\mathbf{a}, \mathbf{f}}$ is in fact finite, thus the number of exceptions in Theorem 2.7 is finite.

2.3. Greatest common divisors of binary linear recurrence sequences. We note that the finiteness conclusion in Question 2.6 would imply that there are finitely many $\alpha \in \overline{\mathbb{Q}}$ such that

$$
(X-\alpha) \mid \operatorname{gcd}\left(F_{n}(X), G_{m}(X)\right)
$$

for some $n, m \geqslant 1$, where by the greatest common divisor of two rational functions, we mean the greatest common divisor of their numerators.

Studying the greatest common divisor of sequences of polynomials has been initiated in [1] for sequences $F_{n}=f^{n}-1$ and $G_{m}=g^{m}-1$, $n, m \geqslant 1$, where $f, g \in \mathbb{C}[X]$, and recently extended in several directions in $[16,26,27,31,32]$.

We note that in the case of simple binary linear recurrence sequences defined over $\mathbb{C}(X)$, under a multiplicative independence condition, a 
finiteness result follows directly from [31, Theorem 1.3]. In fact here we consider a more general scenario, which however holds only when the rational functions involved are defined over $\overline{\mathbb{Q}}$.

More precisely, for a finitely generated group $\Gamma \subseteq \overline{\mathbb{Q}}^{*}$, we define the division group of $\Gamma$ to be the set

$$
\Gamma^{\operatorname{div}}=\left\{\alpha \in \overline{\mathbb{Q}}: \alpha^{n} \in \Gamma \text { for some } n \geqslant 1\right\} .
$$

Then we have the following result.

We say that $\psi_{1}, \ldots, \psi_{t} \in \mathbb{C}(X)$ are multiplicatively independent modulo a set $\mathcal{S} \subseteq \mathbb{C}$ if for all non-zero vectors $\left(h_{1}, \ldots, h_{t}\right) \in \mathbb{Z}^{t}$ one has

$$
\psi_{1}(X)^{h_{1}} \ldots \psi_{t}(X)^{h_{t}} \notin \mathcal{S} .
$$

Theorem 2.9. Let $\Gamma \subseteq \overline{\mathbb{Q}}^{*}$ be a finitely generated subgroup. Let $f_{1}, \ldots, f_{r}, g_{1}, \ldots, g_{s} \in \overline{\mathbb{Q}}(X)$ be multiplicatively independent modulo $\Gamma$. There exists a polynomial $h \in \overline{\mathbb{Q}}[X]$ depending only on $f_{i}, g_{j}$ and the generators of $\Gamma$ such that that for any nonzero vectors $\left(m_{1}, \ldots, m_{r}\right) \in$ $\mathbb{N}^{r},\left(n_{1}, \ldots, n_{s}\right) \in \mathbb{N}^{s}$ and any $u, v \in \Gamma^{\operatorname{div}}$ one has

$$
\operatorname{gcd}\left(\prod_{i=1}^{r} f_{i}(X)^{m_{i}}-u, \prod_{j=1}^{s} g_{j}(X)^{n_{j}}-v\right) \mid h .
$$

Remark 2.10. Taking $r=s=2, \Gamma=\{1\}, m_{1}=n_{1}=1$ and $u=v=$ -1 in Theorem 2.9, one has the sequences $\left(F_{n}\right)_{n=1}^{\infty},\left(G_{m}\right)_{n=1}^{\infty}$ defined by

$$
\begin{aligned}
& F_{n}(X)=f_{1}(X) f_{2}(X)^{n}+1, \quad n \geqslant 1, \\
& G_{m}(X)=g_{1}(X) g\left({ }_{2} X\right)^{m}+1, \quad m \geqslant 1 .
\end{aligned}
$$

In this case the set $\mathcal{L}\left(F_{n}, G_{m}\right)$, defined by (2.4), is finite and its cardinality is uniformly bounded only in terms of the functions $f_{1}, f_{2}, g_{1}, g_{2}$. In fact, this conclusion holds also when $f_{i}, g_{i} \in \mathbb{C}(X), i=1,2$. Indeed, the proof follows directly from [31, Theorem 1.3], which is the same as the proof of Theorem 2.9 but instead of using Lemma 3.2 below, one uses [10, Theorem] (which in turn generalises [9, Theorem 2]). See also [7] for some effective results for rational functions over $\mathbb{Q}$ and $G_{m}=X^{m}-1$.

As a direct consequence of Theorem 2.9 we have the following result, which we hope to be of independent interest.

Corollary 2.11. Let $\Gamma \subseteq \overline{\mathbb{Q}}^{*}$ be a finitely generated subgroup. Let $f_{1}, \ldots, f_{r}, g_{1}, \ldots, g_{s} \in \overline{\mathbb{Q}}(X)$ be multiplicatively independent modulo $\Gamma$. Then there exists a polynomial $H \in \overline{\mathbb{Q}}[X]$ such that for any nonzero vectors $\left(m_{1}, \ldots, m_{r}\right) \in \mathbb{N}^{r},\left(n_{1}, \ldots, n_{s}\right) \in \mathbb{N}^{s}$ and any polynomials 
$F, G \in \overline{\mathbb{Q}}[X]$ of degree at most $d \geqslant 1$ with roots from $\Gamma^{\text {div }}$, one has

$$
\operatorname{gcd}\left(F\left(\prod_{i=1}^{r} f_{i}(X)^{m_{i}}\right), G\left(\prod_{j=1}^{s} g_{j}(X)^{n_{j}}\right)\right) \mid H .
$$

2.4. Specialisations of sequences of rational functions. Finally, we show that a sequence of rational functions $F_{n}(X) \in \mathbb{C}(X), n \geqslant 0$, satisfies a linear recurrence relation if and only if this is also true for specialisations $F_{n}(\alpha), n \geqslant 0$, for a sufficiently "massive" set of $\alpha \in \mathbb{C}$.

Theorem 2.12. Let $\left(F_{n}\right)_{n=0}^{\infty}$ be an infinite sequence of rational functions $F_{n} \in \mathbb{C}(X), n \geqslant 0$, and let $K \geqslant 1$. Assume there exist infinitely many $\alpha \in \mathbb{C}$ such that each $F_{n}(\alpha)$ is well defined and $\left(F_{n}(\alpha)\right)_{n=0}^{\infty}$ is a linear recurrence of order at most $K$. Then $\left(F_{n}\right)_{n=0}^{\infty}$ is a linear recurrence sequence over $\mathbb{C}(X)$ of order at most $K$.

Theorem 2.12 implies that if $\left(F_{n}(\alpha)\right)_{n=0}^{\infty}$ is a linear recurrence sequence for uncountably many $\alpha \in \mathbb{C}$ (without any restriction on the order), then $\left(F_{n}\right)_{n=0}^{\infty}$ is a linear recurrence sequence in $\mathbb{C}[X]$. Indeed, since the set of possible orders is countable, at least one order must occur for infinitely many $\alpha \in \mathbb{C}$ (in fact uncountably many $\alpha$ ). On the other hand, just a condition of the infinitude of such $\alpha \in \mathbb{C}$ is not sufficient. For example, the sequence

$$
F_{n}(X)=X^{2^{n^{2}+n}}+X^{6^{n}}+X^{2^{\lfloor\sqrt{n}\rfloor}}+3^{n}, \quad n=0,1, \ldots,
$$

does not satisfy any linear recurrent relation (as the degree grows too fast). However it is a linear recurrence sequence for specialisations at any root of unity of order $2^{m}, m=0,1, \ldots$

\section{Preliminaries}

3.1. Intersections of level curves of rational functions. Our approach depends on a generalisation of a result of Ailon and Rudnick [1, Theorem 1] which is given in [32, Theorem 2.2].

Recall the definition of non-exceptional pairs of rational functions, that is, pairs which do not satisfy relations of the form (2.2).

Lemma 3.1. Let $g_{1}(X), g_{2}(X) \in \mathbb{C}(X)$ be complex rational functions of degrees $n_{1}$ and $n_{2}$, respectively. Then

$$
\#\left\{z \in \mathbb{C}:\left|g_{1}(z)\right|=\left|g_{2}(z)\right|=1\right\} \leqslant\left(n_{1}+n_{2}\right)^{2},
$$

unless $\left(g_{1}(X), g_{2}(X)\right)$ is exceptional. 
As we have mentioned if $g_{1}$ and $g_{2}$ are polynomials then the conclusion of Lemma 3.1 holds except when

$$
g_{1}=g^{m_{1}} \quad \text { and } \quad g_{2}=g^{m_{2}}
$$

for some $g \in \mathbb{C}[X]$ and integers $m_{1}, m_{2} \geqslant 0$.

\subsection{Intersection of parametric curves with division groups.}

The proof of Theorem 2.9 is based on the following result which is a direct consequence of Maurin's result [30, Théorème 1.2]; see also [8] for an effective proof of [30, Théorème 1.2].

Lemma 3.2. Let $\Gamma \subseteq \overline{\mathbb{Q}}^{*}$ be a finitely generated subgroup. Let $f_{1}, \ldots$, $f_{n} \in \overline{\mathbb{Q}}(X)$ be multiplicatively independent modulo $\Gamma$. Then, there are only finitely many $\alpha \in \overline{\mathbb{Q}}$ such that there exist two linearly independent vectors $\left(k_{1}, \ldots, k_{n}\right),\left(\ell_{1}, \ldots, \ell_{n}\right) \in \mathbb{Z}^{n}$ such that

$$
f_{1}(\alpha)^{k_{1}} \cdots f_{n}(\alpha)^{k_{n}}, f_{1}(\alpha)^{\ell_{1}} \cdots f_{n}(\alpha)^{\ell_{n}} \in \Gamma^{\operatorname{div}} .
$$

Proof. The proof follows directly from a result of Maurin [30, Théorème 1.2] (see also [8, Theorem (Maurin)]). Indeed, let $\gamma_{1}, \ldots, \gamma_{r} \in \Gamma$ be the generators of the group $\Gamma$, and thus, they are multiplicatively independent elements. We define the parametric curve

$$
\mathcal{C}=\left\{\left(f_{1}(\alpha), \ldots, f_{n}(\alpha), \gamma_{1}, \ldots, \gamma_{r}\right): \alpha \in \overline{\mathbb{Q}}\right\} \subset \mathbb{G}_{\mathrm{m}}^{n+r}
$$

For an element $\alpha \in \overline{\mathbb{Q}}$, assume that there exist two linearly independent vectors $\left(k_{1}, \ldots, k_{n}\right),\left(\ell_{1}, \ldots, \ell_{n}\right) \in \mathbb{Z}^{n}$ such that both

$$
f_{1}(\alpha)^{k_{1}} \cdots f_{n}(\alpha)^{k_{n}}, f_{1}(\alpha)^{\ell_{1}} \cdots f_{n}(\alpha)^{\ell_{n}} \in \Gamma^{\mathrm{div}} .
$$

Then, this leads to two multiplicative relations:

$$
\begin{aligned}
& f_{1}(\alpha)^{s k_{1}} \cdots f_{n}(\alpha)^{s k_{n}} \gamma_{1}^{k_{n+1}} \cdots \gamma_{r}^{k_{n+r}}=1 \\
& f_{1}(\alpha)^{t \ell_{1}} \cdots f_{n}(\alpha)^{t \ell_{n}} \gamma_{1}^{\ell_{n+1}} \cdots \gamma_{r}^{\ell_{n+r}}=1
\end{aligned}
$$

for some integer vectors $\left(k_{n+1}, \ldots, k_{n+r}\right),\left(\ell_{n+1}, \ldots, \ell_{n+r}\right) \in \mathbb{Z}^{n+r}$ and some positive integers $s, t$.

Since $f_{1}, \ldots, f_{n}$ are multiplicatively independent modulo $\Gamma$, we know that the functions $f_{1}, \ldots, f_{n}, \gamma_{1}, \ldots, \gamma_{r}$ are multiplicatively independent. We also note that the two vectors $\left(s k_{1}, \ldots, s k_{n}, k_{n+1}, \ldots, k_{n+r}\right)$, $\left(t \ell_{1}, \ldots, t \ell_{n}, \ell_{n+1}, \ldots \ell_{n+r}\right)$ remain linearly independent. Then, the conclusion follows directly from [30, Théorème 1.2]. 
3.3. Zeros of linear recurrence sequences with at most two dominant roots. We say that a root $\lambda$ of a polynomial $\psi(Z) \in \mathbb{C}[Z]$ is dominant if $|\lambda| \geqslant|\rho|$ for any other root $\rho$ of $\psi$. Clearly $\psi$ may have up to $\operatorname{deg} \psi$ dominant roots. We are mostly interested in the case of one or two dominant roots.

We note the following result which represents a simplified combination of two results (for $\nu=1$ and $\nu=2$ ) of Sha [33, Theorems 1.1 and 1.2].

Lemma 3.3. Let $\psi(X) \in \overline{\mathbb{Q}}[X]$ be a monic square-free polynomial of degree $k$ and with $\nu \leqslant 2$ dominant roots. If $\nu=2$, we also impose that the ratio of the two dominant roots is not a root of unity. Then for any linear recurrence sequence $\left(u_{n}\right)_{n=0}^{\infty}$ given by

$$
u_{n}=\sum_{i=1}^{k} \alpha_{i} \lambda_{i}^{n}, \quad n \geqslant 0,
$$

with characteristic polynomial $\psi$ having roots $\lambda_{i}, i=1, \ldots, k$, if

$$
u_{n}=0
$$

then

$$
n \leqslant \exp \left(C_{1} D^{4}\left(\max _{i=1, \ldots, k} h\left(\alpha_{i}\right)+1\right)\right)
$$

where $C_{1}$ is an effective constant which depends only on $k, D=[\mathbb{K}: \mathbb{Q}]$ is the degree of the smallest Galois field $\mathbb{K}$ containing the coefficients of $\psi$ and the initial values $u_{0}, \ldots, u_{k-1}$.

3.4. Zeros of linear recurrence sequences in families. We now recall a special case of a result of Amoroso, Masser and Zannier [2, Theorem 1.5].

Lemma 3.4. Let $f_{i} \in \overline{\mathbb{Q}}(X), i=1, \ldots, k$, be nonzero rational functions such that $f_{s} / f_{r}$ is non-constant for any $1 \leqslant r<s \leqslant k$. There exists an effectively computable constant $C_{2}$, which depends on $f_{1}, \ldots, f_{k}$ such that if for any $b_{1}, \ldots, b_{k} \in \overline{\mathbb{Q}}$ not all zero, any $n \geqslant C_{2}$ and any $\alpha \in$ $\overline{\mathbb{Q}} \backslash \mathfrak{E}_{\mathbf{a}, \mathbf{f}}$, one has

$$
\sum_{i=1}^{k} b_{i} f_{i}(\alpha)^{n}=0
$$

then

$$
h(\alpha) \leqslant \frac{k \max \left\{h\left(b_{1}\right), \ldots, h\left(b_{k}\right)\right\}}{n}+C_{2} .
$$

We remark that in $[2$, Theorem 1.5] the bound is given in terms of the height of the projective vector $\left(b_{1}: \ldots: b_{k}\right) \in \mathbb{P}^{k-1}(\overline{\mathbb{Q}})$ which is 
bounded by the maximum used in Lemma 3.4. Furthermore, in [2, Theorem 1.5] only one ratio is assumed to be non-constant, however there is an additional request of non-vanishing of subsums in the sum of Lemma 3.4 (under our condition on $f_{s} / f_{r}$ one can simply consider the shortest vanishing subsum).

We also note that the effectiveness is not explicitly stated in $[2$, Theorem 1.5] however it is discussed after the formulation of [2, Theorem 1.5].

Corollary 3.5. Let $a_{i}, f_{i} \in \overline{\mathbb{Q}}(X), i=1, \ldots, k$, be nonzero rational functions such that $f_{s} / f_{r}$ is non-constant for any $1 \leqslant r<s \leqslant k$. There exists an effectively computable constant $C_{3}$, which depends on $a_{1}, \ldots, a_{k}, f_{1}, \ldots, f_{k}$ such that if for any $n \geqslant C_{3}$ and any $\alpha \in \overline{\mathbb{Q}}$ one has

$$
\sum_{i=1}^{k} a_{i}(\alpha) f_{i}(\alpha)^{n}=0,
$$

then

$$
h(\alpha) \leqslant C_{3}
$$

Proof. If $\alpha \in \mathfrak{E}_{\mathbf{a}, \mathbf{f}}$, as we have mentioned, this follows from [36, Theorem 3.11].

We can now assume that $\alpha \in \overline{\mathbb{Q}} \backslash \mathfrak{E}_{\mathbf{a}, \mathbf{f}}$. We can also assume that $n>C_{2}$, where $C_{2}$ is as in Lemma 3.4, since otherwise $\alpha$ is a root of an equation of bounded degree and height.

We now recall that for any $\alpha \in \overline{\mathbb{Q}}$ and $a \in \overline{\mathbb{Q}}(X)$ we have

$$
h(a(\alpha)) \leqslant h(\alpha) \operatorname{deg} a+C_{4},
$$

where $C_{4}$ is an effectively computable constant that depends only on $a$, see [36, Theorem 3.11]. Hence for

$$
n \geqslant 2 k \max \left\{\operatorname{deg} a_{1}, \ldots, \operatorname{deg} a_{k}\right\}
$$

the bound of Lemma 3.4 becomes

$$
\begin{aligned}
h(\alpha) & \leqslant \frac{k \max \left\{h\left(\left(a_{1}(\alpha)\right), \ldots, h\left(a_{k}(\alpha)\right)\right\}\right.}{n}+C_{2} \\
& \leqslant \frac{k \max \left\{\operatorname{deg} a_{1}, \ldots, \operatorname{deg} a_{k}\right\} h(\alpha)+k C_{4}}{n}+C_{2} \\
& \leqslant \frac{1}{2} h(\alpha)+C_{4} / 2+C_{2} .
\end{aligned}
$$

Hence $h(\alpha)$ is bounded only in terms of $a_{1}, f_{1}, \ldots, a_{k}, f_{k}$. Choosing

$$
C_{3}=2 k \max \left\{\operatorname{deg} a_{1}, \ldots, \operatorname{deg} a_{k}, C_{2}\right\},
$$

the result follows. 
3.5. Polynomial ABC theorem. We need the following generalisation [11, Theorem 12.4.4] of the ABC Theorem for polynomials proved first by Stothers [39], and then independently by Mason [29] and Silverman [35]. This is a special case of a more general result for $S$-units in function fields due to Voloch [40] and then also Brownawell and Masser [13].

Lemma 3.6. Let $g_{1}, \ldots, g_{m} \in \overline{\mathbb{Q}}[X], m \geqslant 3$, be such that $g_{1}, \ldots, g_{m}$ have no common zero in $\overline{\mathbb{Q}}$. Assume that

$$
g_{1}(X)+\cdots+g_{m}(X)=0
$$

and also that no proper subsum

$$
\sum_{i \in \mathcal{I}} g_{i}(X), \quad \text { with } \quad \mathcal{I} \subseteq\{1, \ldots, m\}, 1 \leqslant \# \mathcal{I}<m,
$$

vanishes identically. Then

$$
\max _{i=1, \ldots, m} \operatorname{deg} g_{i} \leqslant \frac{(m-1)(m-2)}{2} \max \left\{\operatorname{deg} \operatorname{rad}\left(\prod_{i=1}^{m} g_{i}\right)-1,0\right\} .
$$

We also need the following result on the multiplicity of roots of certain rational functions, see [31, Corollary 2.10]. For a rational function $h \in \mathbb{C}(T)$, we denote by $\operatorname{Mult}(h)$ the largest multiplicity of the zeros of $h$ and by $Z(h)$ the set of zeros of $h$ in $\mathbb{C}$, respectively.

Lemma 3.7. Let $h_{i}=f_{i} / g_{i}, f_{i}, g_{i} \in \mathbb{C}[T], i=1, \ldots, n$, with

$$
Z\left(f_{1} \cdots f_{m}\right) \cap Z\left(g_{1} \cdots g_{m}\right)=\varnothing .
$$

Then, for all integers $n_{1}, \ldots, n_{\ell} \geqslant 0$ and $a \in \mathbb{C}^{*}$, we have

$$
\operatorname{Mult}\left(h_{1}^{n_{1}} \cdots h_{\ell}^{n_{m}}-a\right) \leqslant \sum_{i=1}^{m}\left(\operatorname{deg} f_{i}+\operatorname{deg} g_{i}\right) .
$$

3.6. Zeros of linear recurrence sequences in function fields. It is well known that the Skolem Problem is settled in the case of linear recurrence sequences over function fields. An effective (but not explicit) bound on the largest zero in such a sequence is given in [21, Corollary 3.1]. We present it in a simplified form as needed for our setting.

Lemma 3.8. Let $a_{i}, f_{i} \in \overline{\mathbb{Q}}[X], i=1, \ldots, k$, be such that $f_{i} / f_{j} \notin \overline{\mathbb{Q}}^{*}$ for all $1 \leqslant i<j \leqslant k$. Let polynomials $F_{n}, n \geqslant 1$, be defined by (1.2). Then there exists an effectively computable constant $C_{5}$ depending on $a_{i}, f_{i}, i=1, \ldots, k$, such that if $F_{n}=0$ then $n<C_{5}$.

We also have the following lower bound on the number of distinct zeros of $F_{n}(X)$ in (1.2). 
Lemma 3.9. Let $a_{i}, f_{i} \in \overline{\mathbb{Q}}[X], i=1, \ldots, k$, be as in Theorem 2.1 and such that $\operatorname{gcd}\left(a_{1} f_{1}, \ldots, a_{k} f_{k}\right)=1$. Then there exists an effective positive constant $C_{6}$ depending only on $a_{i}, f_{i}, i=1, \ldots, k$, such that for any $n \geqslant C_{6}$, the polynomial $F_{n}$ defined by (1.2) has at least

$$
\frac{2}{k(k-1)} \max _{i=1, \ldots, n}\left\{\operatorname{deg} a_{i}+n \operatorname{deg} f_{i}\right\}-2 d k
$$

distinct roots.

Proof. Let $C_{5}$ be as in Lemma 3.8. Then, for any $n \geqslant C_{5}$ we know that $F_{n} \neq 0$. We consider now the equation

$$
F_{n}(X)-\sum_{i=1}^{k} a_{i}(X) f_{i}(X)^{n}=0 .
$$

We also note that for $n \geqslant C_{5}$ no subsum of the terms from the set

$$
\left\{F_{n}(X), a_{1}(X) f_{1}(X)^{n}, \ldots, a_{k}(X) f_{k}(X)^{n}\right\}
$$

vanishes identically. Indeed, if this happens, then there is a proper subset $\mathcal{I} \subseteq\{1, \ldots, k\}$ such that

$$
\sum_{i \in \mathcal{I}} a_{i}(X) f_{i}(X)^{n}=0 .
$$

Since $n \geqslant C_{5}$, this contradicts Lemma 3.8.

Let $\mathcal{S}_{n}$ be the set of all distinct zeros of $F_{n}$. We can now apply Lemma 3.6 to (3.1) to conclude that

$$
\max _{i=1, \ldots, k}\left\{\operatorname{deg} a_{i}+n \operatorname{deg} f_{i}\right\} \leqslant \frac{k(k-1)}{2}\left(\# \mathcal{S}_{n}+\sum_{i=1}^{k}\left(\operatorname{deg} a_{i}+\operatorname{deg} f_{i}\right)\right) .
$$

From here we obtain

$$
\# \mathcal{S}_{n} \geqslant \frac{2 \max _{i=1, \ldots, k}\left\{\operatorname{deg} a_{i}+n \operatorname{deg} f_{i}\right\}}{k(k-1)}-2 d k,
$$

which concludes the proof (with $C_{6}=C_{5}$ ).

Remark 3.10. We note that under a more restrictive condition that the $k$ products $a_{1} f_{1}, \ldots, a_{k} f_{k}$ are pairwise relatively prime, one can take $C_{6}=1$ in Lemma 3.9. Furthermore, under the more restrictive condition that $2 k$ polynomials $a_{1}, f_{1}, \ldots, a_{k}, f_{k}$ are pairwise relatively prime, one can also use the result of Brindza [12, Theorem 1] to show that the polynomial $F_{n}$ has at least $n /(k-1)-(d+1) k$ distinct roots.

We also remark that a tight lower bound for the degree of $F_{n}$ has recently been given in [20, Corollary 2] (in fact for arbitrary nondegenerate linear recurrences including non-simple ones). 
3.7. Characterisation of linear recurrence sequences. To test whether an arbitrary sequence $\left(v_{n}\right)_{n=1}^{\infty}$ of elements of a field $\mathbb{F}$ is a linear recurrence sequence, we recall the following well-known result which is based on the vanishing of the Kronecker-Hankel determinants

$$
\Delta_{h}=\operatorname{det}\left(v_{i+j}\right)_{0 \leqslant i, j \leqslant h-1} .
$$

More precisely, by [28, Theorem 8.75] (see also [18, Theorem 1.6] and [23, Lemma 5, Chapter V] for variations) we have:

Lemma 3.11. A sequence $\left(v_{n}\right)_{n=1}^{\infty}$ of elements of a field $\mathbb{F}$ is a linear recurrence sequence of order $k$ if and only if for the determinants (3.2) we have $\Delta_{h}=0$ for all $h \geqslant k+1$.

\section{Proofs of Results Towards the Skolem Problem}

4.1. Proof of Theorem 2.1. Multiplying by common denominators of $a_{i}(X)$ and of $f_{i}(X), i=1, \ldots, k$, we can assume that $a_{i}(X), f_{i}(X) \in$ $\overline{\mathbb{Q}}[X], i=1, \ldots, k$.

Let $\alpha \in \overline{\mathbb{Q}} \backslash \mathfrak{E}_{\mathbf{a}, \mathbf{f}}$ be such that (2.3) holds.

We now consider the case when we have at least three dominant roots, that is, there exist distinct integers $1 \leqslant r<s<t \leqslant k$ such that

$$
\left|f_{r}(\alpha)\right|=\left|f_{s}(\alpha)\right|=\left|f_{t}(\alpha)\right|
$$

or equivalently,

$$
\frac{\left|f_{s}(\alpha)\right|}{\left|f_{r}(\alpha)\right|}=\frac{\left|f_{t}(\alpha)\right|}{\left|f_{r}(\alpha)\right|}=1 .
$$

Since by hypothesis, $\left(f_{s} / f_{r}, f_{t} / f_{r}\right)$ is a non-exceptional rational function, from Lemma 3.1 we see that for each of the $k(k-1)(k-2) / 6$ possible choices of the triple $(r, s, t)$ there are at most $4 d^{2}$ such $\alpha \in \overline{\mathbb{Q}}$.

Hence in total we have excluded at most

$$
4 d^{2} k(k-1)(k-2) / 6=2 d^{2} k(k-1)(k-2) / 3
$$

elements $\alpha \in \overline{\mathbb{Q}} \backslash \mathfrak{E}_{\mathbf{a}, \mathbf{f}}$.

We thus assume that we have at most two dominant roots. In this case we apply Lemma 3.3 to any element $\alpha \in \overline{\mathbb{Q}} \backslash \mathfrak{E}_{\mathbf{a}, \mathbf{f}}$ and derive that

$$
n \leqslant \exp \left(C_{7} D_{\alpha}^{4}(h(\alpha)+1)\right)
$$

for an effectively computable constant $C_{7}$, which depends only on $a_{1}, \ldots, a_{k}, f_{1}, \ldots, f_{k}$. We now observe that for $n \leqslant C_{3}$, where $C_{3}$ is as in Corollary 3.5, the result is trivial. Otherwise Corollary 3.5 applies and we obtain the desired result. 
4.2. Proof of Corollary 2.3. By Theorem 2.1 we only need to estimate the number $R(D)$ of roots of unity of degree at most $D$ over $\mathbb{Q}$. Clearly

$$
R(D)=\sum_{m: \varphi(m) \leqslant D} \varphi(m) \leqslant D \sum_{m: \varphi(m) \leqslant D} 1,
$$

where $\varphi(m)$ is the Euler function. Using the bound

$$
\sum_{m: \varphi(m) \leqslant D} 1 \leqslant 23 D
$$

of Dubickas and Sha [17, Lemma 4.1], we see that

$$
R(D) \leqslant 23 D^{2}
$$

(using partial summation one can certainly obtain a tighter bound). Now, since each of $k(k-1) / 2$ ratios $f_{i}(\alpha) / f_{j}(\alpha)$ can be a root of unity of degree at most $D$, there are at most

$$
d R(D) \frac{k(k-1)}{2}<12 d D^{2} k^{2}
$$

such $\alpha \in \mathcal{A}_{D}$, which we need to exclude.

Taking into account that we also need to exclude at most other $2 d^{2} k(k-1)(k-2) / 3$ elements $\alpha \in \mathcal{A}_{D}$ as in Theorem 2.1, and at most $2 d k$ zeros of $a_{i} f_{i}, i=1, \ldots, k$, and thus at most

$$
2 d^{2} k(k-1)(k-2) / 3+2 d k \leqslant 2 d^{2} k^{3} / 3
$$

elements (elementary calculus show that the last inequality holds for any $d \geqslant 1$ and $k \geqslant 2$ ). Hence we conclude the proof.

4.3. Proof of Corollary 2.4. Let $\mathbb{K}$ be a number field with $[\mathbb{K}: \mathbb{Q}]=$ $D$ such that $a_{i}, f_{i} \in \mathbb{K}[X], i=1, \ldots, k$.

Let $\alpha \notin \mathfrak{E}_{\mathbf{a}, \mathbf{f}}$ be a root of $F_{n}$ for some $n \geqslant 1$. Let $G$ be its minimal polynomial over $\mathbb{K}$, which implies that $G \mid F_{n}$. Let $D_{G}=\operatorname{deg} G=$ $[\mathbb{K}(\alpha): \mathbb{K}]$ and let $\mathbb{M}_{\alpha}$ be the smallest Galois extension of $\mathbb{Q}$ which includes $\mathbb{K}(\alpha)$.

For any $\sigma \in \operatorname{Gal}(\mathbb{K}(\alpha) / \mathbb{K})$, conjugating the relation $F_{n}(\alpha)=0$ gives us

$$
\sum_{i=1}^{k} a_{i}(\sigma(\alpha)) f_{i}(\sigma(\alpha))^{n}=0 .
$$

We may assume that $D_{G}>2 d^{2} k^{3} / 3$. Indeed, if $D_{G} \leqslant 2 d^{2} k^{3} / 3$, then

$$
[\mathbb{K}(\alpha): \mathbb{Q}] \leqslant D D_{G} \leqslant \frac{2}{3} D d^{2} k^{3}
$$

and since by Corollary 3.5, $h(\alpha)$ is bounded from the above by a constant depending only on $a_{1}, f_{1}, \ldots, a_{k}, f_{k}$, by Northcott's Theorem 
there are finitely many such $\alpha$. Thus we can exclude the irreducible factors of $F_{n}, n \geqslant 1$, corresponding to these finitely many elements.

Now, since there are $D_{G}>2 d^{2} k^{3} / 3$ distinct elements $\sigma(\alpha)$ satisfying (4.3), and taking into account the hypothesis on the polynomials $f_{i}$, we can apply Theorem 2.1 to conclude that there exists $\sigma \in \operatorname{Gal}(\mathbb{K}(\alpha) / \mathbb{K})$ such that (4.3) holds and for which

$$
n \leqslant \exp \left(C_{8}\left[\mathbb{M}_{\alpha}: \mathbb{Q}\right]^{4}\right)
$$

for some constant $C_{8}$ which depends only on $a_{1}, f_{1}, \ldots, a_{k}, f_{k}$. This concludes the proof.

4.4. Proof of Corollary 2.5. Let the set $\mathcal{E}$ be as in Corollary 2.4. Clearly, if $F_{n}$ has a root outside of the set $\mathcal{E} \cup \mathfrak{E}_{\mathrm{a}, \mathrm{f}}$ the result is instant from Corollary 2.4.

Otherwise, since $\mathcal{E}$ is a finite set and so are the sets of $\alpha \in \overline{\mathbb{Q}}$ with $f_{i}(\alpha)=0$ or $a_{i}(\alpha)=0$, for some $1 \leqslant i \leqslant k$, adjusting the constant $c_{0}$ we can make the desired result valid for these values of $\alpha$ as well.

Hence it remains to consider the case when the ratio $f_{i}(\alpha) / f_{j}(\alpha)$ is a root of unity for some $1 \leqslant i<j \leqslant k$. We recall that by Lemma 3.9 $F_{n}$ has at least $2 n / k(k-1)-C_{9}$ distinct roots $\alpha$, for some constant $C_{9}$ which depends only on $a_{1}, f_{1}, \ldots, a_{k}, f_{k}$. By our assumption, for each of them at least one ratio $f_{i}(\alpha) / f_{j}(\alpha)$ is a root of unity for some $1 \leqslant i<j \leqslant k$. For each $\gamma \in \overline{\mathbb{Q}}$ the equation $f_{i}(\alpha) / f_{j}(\alpha)=\gamma$ has at most $d$ solutions $\alpha \in \overline{\mathbb{Q}}$. Hence the set of roots of unity among the ratios $f_{i}(\alpha) / f_{j}(\alpha)$ form a set $\mathcal{U}_{\mathbf{f}}$ of at least

$$
\# \mathcal{U}_{\mathbf{f}} \geqslant 2 n / d k(k-1)-C_{9} / d
$$

distinct roots of unity $\rho$ for some $1 \leqslant i<j \leqslant k$.

Recall the bound (4.2) on the number of roots of unity of degree at most $D$. Hence at least one of the above roots of unity is of degree at least

$$
\sqrt{\frac{1}{23} \# \mathcal{U}_{\mathbf{f}}} \geqslant \sqrt{\frac{2 n}{23 d k(k-1)}-C_{9} / 23 d} .
$$

and thus so is $\alpha$. Hence in this case we have a much stronger bound than claimed.

\section{Proof of Theorem 2.7 on Perfect Powers in Specialisations at Roots of Unity}

Let $\mathbb{K}$ be the field of definition of the polynomials $a_{i}, f_{i}$. Thus, $a_{i}, f_{i} \in$ $\mathbb{Z}_{\mathbb{K}}[X]$, where $\mathbb{Z}_{\mathbb{K}}$ is the ring of integers of $\mathbb{K}$. 
As we have shown in the proof of Theorem 2.1, see (4.1), for all but at most $2 d^{2} k^{3} / 3$ elements $\alpha \in \mathbb{U} \backslash \mathfrak{E}_{\mathbf{a}, \mathbf{f}}$, the sequence $\left(F_{n}(\alpha)\right)_{n=1}^{\infty}$ has at most two dominant roots.

Let us assume first that for such an element $\alpha \in \mathbb{U} \backslash \mathfrak{E}_{\mathbf{a}, \mathbf{f}}$, the sequence $\left(F_{n}(\alpha)\right)_{n=1}^{\infty}$ has two dominant roots, that is, for some $1 \leqslant i \neq j \leqslant k$, $\left|f_{i}(\alpha)\right|=\left|f_{j}(\alpha)\right|$. This implies that

$$
f_{i}(\alpha) \overline{f_{i}}(\bar{\alpha})=f_{i}(\alpha) \overline{f_{i}(\alpha)}=f_{j}(\alpha) \overline{f_{j}(\alpha)}=f_{j}(\alpha) \overline{f_{j}}(\bar{\alpha}),
$$

where $\bar{\alpha}$ is the complex conjugate of $\alpha$, which is again a root of unity.

Since by our assumption,

$$
f_{i}(X) \overline{f_{i}}(Y)-f_{j}(X) \overline{f_{j}}(Y) \in \mathbb{K}[X, Y], \quad 1 \leqslant i \neq j \leqslant k,
$$

do not have any factor of the form $X^{r} Y^{s}-u$ or $X^{r}-u Y^{s}$ with $u \in \mathbb{U}$, by a result of Lang [25] we know that there are finitely many solutions $(\alpha, \beta)$ in roots of unity (and thus also of the form $(\alpha, \bar{\alpha})$ ) to the equation (5.1). Moreover, by [6, Section 4.1], the equation

$$
f_{i}(X) \overline{f_{i}}(Y)-f_{j}(X) \overline{f_{j}}(Y)=0
$$

has at most $44 d^{2}$ solutions $(\alpha, \beta)$ in roots of unity. Thus, there are at most $22 d^{2} k(k-1)<22 d^{2} k^{2}$ such elements $\alpha \in \mathbb{U} \backslash \mathfrak{E}_{\mathbf{a}, \mathbf{f}}$ such that the sequence $\left(F_{n}(\alpha)\right)_{n=1}^{\infty}$ has two dominant roots.

Thus, for all but $d^{2}\left(2 k^{3} / 3+22 k^{2}\right)$ elements $\alpha \in \mathbb{U} \backslash \mathfrak{E}_{\mathbf{a}, \mathbf{f}}$, the sequence $\left(F_{n}(\alpha)\right)_{n=1}^{\infty}$ has only one dominant root. Let such an element $\alpha \in$ $\mathbb{U} \backslash \mathfrak{E}_{\mathbf{a}, \mathbf{f}}$. We now apply the result of Fuchs [19, Corollary 2.10] describing the structure of the set of perfect powers in linear recurrent sequences over number fields (one easily verifies that for $\alpha \in \mathbb{U} \backslash \mathfrak{E}_{\mathbf{a}, \mathbf{f}}$ all necessary conditions of $[19$, Corollary 2.10] are satisfied). This concludes the proof.

\section{Proofs of Results on Common Zeros}

6.1. Proof of Theorem 2.9. For given $\mathbf{m}=\left(m_{1}, \ldots, m_{r}\right), \mathbf{n}=$ $\left(n_{1}, \ldots, n_{s}\right)$, and $u, v \in \Gamma^{\text {div }}$, we define

$$
\mathcal{D}_{\mathbf{m}, \mathbf{n}, u, v}(X)=\operatorname{gcd}\left(\prod_{i=1}^{r} f_{i}(X)^{m_{i}}-u, \prod_{j=1}^{s} g_{j}(X)^{n_{j}}-v\right) .
$$

We recall that by the greatest common divisor of two rational functions we mean the greatest monic common divisor of their numerators. In particular, $\mathcal{D}_{\mathbf{m}, \mathbf{n}, u, v} \in \overline{\mathbb{Q}}[X]$.

The proof follows the same approach as in the proof of [31, Theorem 1.3]. Indeed, since the rational functions $f_{1}, \ldots, f_{r}, g_{1}, \ldots, g_{s}$ are 
multiplicatively independent modulo $\Gamma$, by Lemma 3.2, applied with $n=r+s$ and

$$
\begin{aligned}
& \left(k_{1}, \ldots, k_{n}\right)=\left(m_{1}, \ldots, m_{r}, 0, \ldots, 0\right), \\
& \left(\ell_{1}, \ldots, \ell_{n}\right)=\left(0, \ldots, 0, n_{1}, \ldots, n_{s}\right),
\end{aligned}
$$

there are finitely many $\alpha \in \overline{\mathbb{Q}}$ such that $\mathcal{D}_{\mathbf{m}, \mathbf{n}, u, v}(\alpha)=0$ for some nonzero integer vectors $\mathbf{m}, \mathbf{n}$ as above and $u, v \in \Gamma^{\text {div }}$. We denote this finite set, depending only on $f_{i}, g_{j}$, by $\mathcal{S}$.

To construct the polynomial $h$ we need to control also the multiplicity of the roots of the polynomials $\mathcal{D}_{\mathbf{m}, \mathbf{n}, u, v}$. This is also readily given by Lemma 3.7, which implies that

$$
\operatorname{Mult}\left(\mathcal{D}_{\mathbf{m}, \mathbf{n}, u, v}\right) \leqslant 2 \min \left\{\sum_{i=1}^{r} \operatorname{deg} f_{i}, \sum_{j=1}^{s} \operatorname{deg} g_{j}\right\} .
$$

Thus, we can define the polynomial $h \in \overline{\mathbb{Q}}[X]$ by

$$
h(X)=\prod_{\gamma \in \mathcal{S}}(X-\gamma)^{2 \min \left\{\sum_{i=1}^{r} \operatorname{deg} f_{i}, \sum_{j=1}^{s} \operatorname{deg} g_{j}\right\}},
$$

which concludes the proof.

6.2. Proof of Corollary 2.11. Let $F, G \in \overline{\mathbb{Q}}[X]$ be of degree at most $d \geqslant 1$ with all roots in $\Gamma^{\text {div }}$. We reduce the problem to looking at each greatest common divisor

$$
\operatorname{gcd}\left(\prod_{i=1}^{r} f_{i}(X)^{m_{i}}-\gamma_{1}, \prod_{j=1}^{s} g_{j}(X)^{n_{j}}-\gamma_{2}\right)
$$

with roots $\gamma_{1}$ and $\gamma_{2}$ of $F$ and $G$ respectively and $\left(m_{1}, \ldots, m_{r}\right) \in$ $\mathbb{N}^{r} \backslash\{\mathbf{0}\},\left(n_{1}, \ldots, n_{s}\right) \in \mathbb{N}^{s} \backslash\{\mathbf{0}\}$.

By Theorem 2.9 there exists a polynomial $h \in \overline{\mathbb{Q}}[X]$ that depends only on $f_{i}, g_{j}$ and the generators of $\Gamma$ such that for all $\left(m_{1}, \ldots, m_{r}\right) \in$ $\mathbb{N}^{r} \backslash\{\mathbf{0}\},\left(n_{1}, \ldots, n_{s}\right) \in \mathbb{N}^{s} \backslash\{\mathbf{0}\}$ and $\gamma_{1}, \gamma_{2} \in \Gamma^{\text {div }}$ one has

$$
\operatorname{gcd}\left(\prod_{i=1}^{r} f_{i}(X)^{m_{i}}-\gamma_{1}, \prod_{j=1}^{s} g_{j}(X)^{m_{j}}-\gamma_{2}\right) \mid h .
$$

Since both $F$ and $G$ have at most $d$ roots in $\Gamma^{\text {div }}$, we conclude the proof choosing $H=h^{d^{2}}$. 


\section{Proof of Theorem 2.12}

For each $\alpha \in \mathbb{C}$ we consider the sequence $\left(\Delta_{h}(\alpha)\right)_{h=0}^{\infty}$ of KroneckerHankel determinants

$$
\Delta_{h}(\alpha)=\operatorname{det}\left(F_{i+j}(\alpha)\right)_{0 \leqslant i, j \leqslant h-1}, \quad h=0,1, \ldots
$$

Assume there exist infinitely many $\alpha \in \mathbb{C}$ such that $\left(F_{n}(\alpha)\right)_{n=0}^{\infty}$ is a linear recurrence of order at most $K$. Hence, be Lemma 3.11 for all $h \geqslant K+1$ we have

$$
\Delta_{h}(\alpha)=0
$$

for infinitely many $\alpha \in \mathbb{C}$. Therefore, for all $h \geqslant K+1$, the rational function

$$
\Delta_{h}(X)=\operatorname{det}\left(F_{i+j}(X)\right)_{0 \leqslant i, j \leqslant h-1}
$$

has infinitely many zeros, and thus is identical to zero.

Applying Lemma 3.11 again, we conclude the proof.

\section{Comments And Further Questions}

As we have mentioned Theorem 2.1 can be extended to non-simple sequences without new ideas and just at the cost of introducing more complicated notations.

We also remark that Theorem 2.1 is an analogue of a result of Kulkarni, Mavraki, and Nguyen [24, Proposition 2.2], see also [4, Proposition 2.2], in which the coefficients $a_{1}, \ldots, a_{k}$ of $F_{n}(X)$ in (1.2) are constants rather than rational functions as in our case. Moreover, [24, Proposition 2.2] is not effective while Theorem 2.1 is.

Furrhermore, if as in [24] the coefficients $a_{1}, \ldots, a_{k}$ of $F_{n}(X)$ in $(1.2)$ are constants then analysing the bounds of [33] underlying Lemma 3.3 we see that $D^{4}$ can be replaced with $D_{0}^{3} D$, where $D_{0}$ is the degree of the Galois closure of $\mathbb{Q}\left(a_{1}, \ldots, a_{k}\right)$ over $\mathbb{Q}$. In turn, in this case, this leads to a single exponential bound of the form

$$
n \leqslant \exp \left(C D_{\alpha}\right)
$$

in Theorem 2.1.

We describe a possible generalisation of Theorem 2.1 to $S$-unit equations in $\overline{\mathbb{Q}}(X)$. Let $\Gamma$ be a finitely generated subgroup of $\overline{\mathbb{Q}}(X)$ and fix rational functions $a_{1}, \ldots, a_{k} \in \overline{\mathbb{Q}}(X)$. By [2, Proposition 6.1], for any rational functions $u_{1}, \ldots, u_{k} \in \Gamma$ such that

$$
u_{i} / u_{j} \notin \overline{\mathbb{Q}}, \quad 1 \leqslant i<j \leqslant k, \quad \text { and } \quad \sum_{i=1}^{k} a_{i} u_{i} \neq 0
$$


the set of $\alpha \in \overline{\mathbb{Q}}$ such that

$$
\sum_{i=1}^{k} a_{i}(\alpha) u_{i}(\alpha)=0
$$

is a set of bounded height, depending only on $a_{1}, \ldots, a_{k}$ and the generators of $\Gamma$.

We now ask for an analogue of Theorem 2.1 for such equations. It is convenient to define the notion of a primitive solution to (8.1) as a solution with $u_{i}(\alpha)=1$ for some $i=1, \ldots, k$.

We now ask the following.

Question 8.1. Is it true, under some natural conditions on the generators of $\Gamma$, that outside of a set of bounded height of values $\alpha \in \overline{\mathbb{Q}}$, then for every primitive solution to (8.1), $\max _{i=1, \ldots, k} \operatorname{deg} u_{i}$ is bounded only in terms of the degree $D_{\alpha}$ of the smallest Galois field $\mathbb{K}$ over $\mathbb{Q}$ with $\alpha \in \mathbb{K}$, the functions $a_{1}, \ldots, a_{k}$ and the generators of $\Gamma$ ?

We note that the idea of the proof of [2, Proposition 6.1] which reduces $S$-unit equations to equations of the type (2.3), can perhaps help to tackle Question 8.1. Unfortunately, during this reduction we do not control well the corresponding polynomials $a_{1}, f_{1}, \ldots, a_{k}, f_{k} \in \overline{\mathbb{Q}}(X)$ and in particular it is not immediately clear how to verify the necessary condition of Theorem 2.1.

We remark that the proof of Theorem 2.1 relies on the fact that outside of a small set of parameters the corresponding specialisations are sequences with at most two dominant roots. On the other hand, in the proof of Theorem 2.7 we show that for all but finitely many specialisations at roots of unity these sequences have only one dominant root. These ideas can be used to study many other properties of the corresponding linear recurrence sequences. For example, by combining this approach with results and ideas of $[34,37,38]$ one can study prime ideal divisors of elements of these sequences.

\section{ACKNOWLEDGEMENT}

The authors are very grateful to the referee for the very helpful comments.

This work was supported, in part, by the Australian Research Council Grants DP180100201 and DP200100355. The authors are grateful to Umberto Zannier for useful discussions on previous versions of the file. The first author gratefully acknowledges the hospitality and generosity of the Max Planck Institute of Mathematics, where parts of this paper were developed. 


\section{REFERENCES}

[1] N. Ailon and Z. Rudnick, 'Torsion points on curves and common divisors of $a^{k}-1$ and $b^{k}-1$ ', Acta Arith., 113 (2004), 31-38. 3, 7, 9

[2] F. Amoroso, D. Masser and U. Zannier, 'Bounded height in pencils of finitely generated subgroups', Dule Math. J., 166 (2017) 2599-2642. 2, 3, 6, 11, 12, 20, 21

[3] F. Amoroso and E. Viada, 'On the zeros of linear recurrence sequences', Acta Arith., 147 (2011), 387-396. 1

[4] J. P. Bell, K. D. Nguyen and U. Zannier, ' $D$-finiteness, rationality, and height', Trans. Amer. Math. Soc., 373 (2020), 4889-4906. 20

[5] J. Berstel and M. Mignotte, 'Deux propriétés décidables des suites récurrentes linéaires', Bull. Soc. Math. France, 104 (1976), 175-184. 2, 5

[6] F. Beukers and C. J. Smyth, 'Cyclotomic points on curves', Number Theory for the Millenium (Urbana, Illinois, 2000), vol. I, A. K. Peters, 2002, 67-85. 18

[7] Y. Bilu and F. Luca, 'Binary polynomial power sums vanishing at roots of unity', Acta Arith., to appear. 8

[8] E. Bombieri, P. Habegger, D. Masser and U. Zannier, A Note on Maurin Theorem, Rend. Lincei Mat. Appl. 21 (2010), 251-260. 10

[9] E. Bombieri, D. Masser and U. Zannier, 'Intersecting a curve with algebraic subgroups of multiplicative groups', Int. Math. Res. Not., 20 (1999), 11191140. 7,8

[10] E. Bombieri, D. Masser and U. Zannier, 'On unlikely intersections of complex varieties with tori', Acta Arith., 133 (2008), 309-323. 8

[11] E. Bombieri and W. Gubler, Heights in Diophantine geometry, Cambridge Univ. Press, Cambridge, 2006. 3, 4, 13

[12] B. Brindza, 'Zeros of polynomials and exponential diophantine equations', Compos. Math., 61 (1987), 137-157. 14

[13] W. D. Brownawell, D. Masser, 'Vanishing sums in function fields', Math. Proc. Cambridge Philos. Soc., 100 (1986), 427-434. 13

[14] Y. Bugeaud, P. Corvaja and U. Zannier, 'An upper bound for the G.C.D. of $a^{n}-1$ and $b^{n}-1$ ', Math. Z., 243 (2003), 79-84. 3

[15] P. Corvaja and U. Zannier, 'A lower bound for the height of a rational function at $S$-unit points', Monatsh. Math., 144 (2005), 203-224. 3

[16] P. Corvaja and U. Zannier, 'Some cases of Vojta's conjecture on integral points over function fields', J. Alg. Geometry, 17 (2008), 295-333. 3, 7

[17] A. Dubickas and M. Sha, 'The distance to square-free polynomials', Acta Arith., 186 (2018), 243-256. 16

[18] G. Everest, A. J. van der Poorten, I. E. Shparlinski and T. B. Ward, Recurrence sequences, Amer. Math. Soc., 2003. 1, 5, 15

[19] C. Fuchs, 'Polynomial-exponential equations and linear recurrences', Glasnik Matem., 38 (2003), 233-252. 18

[20] C. Fuchs and S. Heintze, 'On the growth of linear recurrences in function fields', Preprint, 2020, available at https://arxiv.org/abs/2006.11074. 14

[21] C. Fuchs, and A. Pethö, 'Effective bounds for the zeros of linear recurrences in function fields', Journal de Théorie des Nombres de Bordeaux, 17 (2005), 749-766. 6, 13

[22] M. Hindry and J. H. Silverman, Diophantine geometry, Graduate Texts in Mathematics 201, Springer, New York, 2000. 4 
[23] N. Koblitz, P-adic numbers, p-adic analysis, and zeta-functions, SpringerVerlag, New York, 1977. 15

[24] A. Kulkarni, N. M. Mavraki, and K. D. Nguyen, 'Algebraic approximations to linear combinations of powers: an extension of results by Mahler and CorvajaZannier', Trans. Amer. Math. Soc., (to appear). 2, 20

[25] S. Lang, 'Division points on curves', Ann. Mat. Pura Appl., 70 (1965), 229234. 7,18

[26] A. Levin, 'Greatest common divisors and Vojta?s conjecture for blowups of algebraic tori', Invent. Math., 215 (2019), no. 2, 493-533. 3, 7

[27] A. Levin and J. T.-Y. Wang, 'Greatest common divisors of analytic functions and Nevanlinna theory of algebraic tori', J. Reine Angew. Math., (to appear). 3,7

[28] R. Lidl and H. Niederreiter, Finite Fields, Cambridge Univ. Press, Cambridge, 1997. 15

[29] R. C. Mason, Diophantine equations over function fields, London Math. Soc. Lecture Note Series 96, Cambridge Univ. Press, Cambridge, 198413

[30] G. Maurin, 'Courbes algébriques et équations multiplicatives', Math. Ann., 341 (2008), 789-824. 10

[31] A. Ostafe, 'On some extensions of the Ailon-Rudnick theorem', Monat. für Math., 181 (2016), 451-471. 3, 7, 8, 13, 18

[32] F. Pakovich and I. E. Shparlinski, 'Level curves of rational functions and unimodular points on rational curves', Proc. Amer. Math. Soc., 148 (2020), 1829 1833. 3, 7, 9

[33] M. Sha, 'Effective results on the Skolem problem for linear recurrence sequences', J. Number Theory, 197 (2019) 228-249. 1, 2, 3, 11, 20

[34] I. E. Shparlinski, 'Prime divisors of recurrent sequences', Isv. Vyssh. Uchebn. Zaved. Math, no.4, (1980), 101-103 21

[35] J. H. Silverman, 'The S-unit equation over function fields', Proc. Camb. Philos. Soc., 95 (1984), 3-4. 13

[36] J. H. Silverman, The arithmetic of dynamical systems, Springer-Verlag, New York, 2007. 4, 5, 12

[37] C. L. Stewart, 'On divisors of terms of linear recurrence sequences', J. Reine Angew. Math., 333 (1982), 12-31. 21

[38] C. L. Stewart, 'On the greatest square-free factor of terms of a linear recurrence sequence', Diophantine Equations Tata Inst. Fund. Res. Stud. Math., 20, Tata Inst. Fund. Res., Mumbai, 2008, 257-264, 21

[39] W. W. Stothers, 'Polynomial identities and Hauptmoduln', Quarterly J. Math. Oxford, 32 (1981), 349-370. 13

[40] J. F. Voloch, 'Diagonal equations over function fields', Bol. Soc. Brasil. Mat., 16 (1985), 29-39. 13

[41] U. Zannier, Lecture notes on Diophantine analysis, Publ. Scuola Normale Superiore, Pisa, 2009. 4 
School of Mathematics and Statistics, University of New South Wales, Sydney NSW 2052, Australia

Email address: alina.ostafe@unsw.edu.au

School of Mathematics and Statistics, University of New South Wales, Sydney NSW 2052, Australia

Email address: igor.shparlinski@unsw.edu.au 